\title{
Contributo alle conoscenze sismo-tettoniche della regione istmica a Sud di Catanzaro
}

\author{
G. M. CAMELI \\ Ricevuto il 27 Aprile 1966
}

\begin{abstract}
Riassunto. - Si studia il mecanismo che ha dato origine alla scossa all'ipocentro del terremoto dell'll Maggio 1947 avvenuto nel Golfo di Squillace e si pongono in relazione i risultati con le caratteristiche gravimetriche e sismo-tettoniche della regione istmica compresa fra il Golfo di Sant'Eufemia e quello di Squillace.
\end{abstract}

SUMmary. - This paper reports the study on the physical nature of the earthquake in Squillace Gulf (5th May 1947).

The results are compared with the gravimetric and seismic-tectonic characteristics of the istlimus between Sant'Eufemia and Squillace Gulfs.

Nel quadro degli studi che si compiono presso l'Istituto Nazionale di Geofisica sulle caratteristiche sismiche della regione Italiana mi sono proposto di esaminare in dettaglio l'istmo che separa il Golfo di Squillace e quello di Sant'Eufemia riprendendo il terremoto già studiato da $P$. E. Valle (') e il eui epicentro è nel Mar Jonio a SE di Squillace.

L'autore perviene alle seguenti coordinate epicentrali:

$$
\begin{aligned}
& \varphi_{o}=38^{\circ} 41^{\prime}, 4 \pm 7^{\prime}, 6 \mathrm{~N} \\
& \lambda_{o}=16^{\circ} 47^{\prime}, 7 \pm 2^{\prime}, 2 \mathrm{E} \\
& H_{o}=06^{\mathrm{n}} 32^{\mathrm{m} 1} 5^{\mathrm{s}}, 4 \pm \mathbf{1}^{\mathrm{s}}, 4 \text { (T.M.G.) }
\end{aligned}
$$

e non assegna una particolare profondità ipocentrale, ritenendo l'ipocentro compreso nella crosta.

Dato che ci si prefigge di indagare sul meccanismo che ha dato origine alla scossa all'ipocentro possiamo ritenere il terremoto superficiale ed applicare ad esso il metodo di Byerly modificato da Di Filippo $\left(^{2}\right)$. 
Come è noto nel caso di terremoti di frattura i due piani ortogonali nell'epicentro dividono la terra in quattro parti di cui due, in quadranti opposti, mostrano come primo impulso compressione e le altre due dilatazione. Dei due piani determinati graficamente uno è il piano di frattura, laltro è il piano secondario. Nei terremoti superficiali si considera l'epicentro coincidente con l'ipocentro, come nel caso del terremoto in esame; pertanto le intersezioni dei due piani con la superficie terrestre risultano circonferenze normali tra di loro e passanti per l'epicentro.

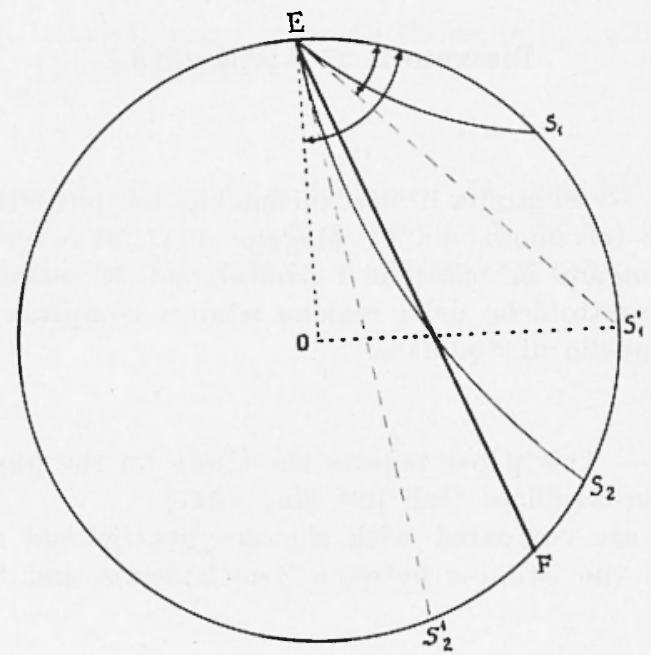

Fig. 1

Se la terra fosse omogenea nelle quattro zone delimitate dai due cerchi il primo impulso sarebbe, all'arrivo nelle stazioni, della stessa natura presentata all'origine. La Terra ì però formata da strati eterogenei e se cio non influisee sulla nauma dell'impulso lungo i] raggio sismico tuttavia obbliga detto raggio secondo ma traiettoria curva; in tal modo il raggrio sismico può far giungere in superficie un impulso di diversa natuma da quello atteso.

Infatti dalla Fig. 1 si nota che, essendo $E$ l'epicent ro, $S_{1}$ ed $S_{2}$ due stazioni sismiche ed $E F$ la traccia dell'eventuale piano di flattura, pur giacendo le due stazioni dalla stessa parte del piano esse registrano impulsi provenienti da zone assai diverse della Terra.

Conoscendo le distanze epicentrali dagli Osservatori e la dromocrona delle longitudinali è determinabile l'angolo di emergenza la cui rotan- 
gente ci permette di ottenere le nuove distanze epicentrali corrispondenti ai raggi considerati rettilinei.

Nella Tabella 1 sono riportati gli elementi necessari a rappresentare le stazioni in proiezione stereografica polare sul piano equatoriale usando come polo di proiezione l'antiepicentro, nonché i primi impulsi

Tabella I

\begin{tabular}{|c|c|c|c|c|c|}
\hline $\mathrm{N}$ & Osservatori & $\begin{array}{l}\text { impulsi } \\
\text { iniziali }\end{array}$ & $\Delta 0$ & Azimut & $\operatorname{cotg} i$ \\
\hline 1 & Catania & + & $1^{\circ} 39^{\prime}$ & $234^{\circ} 40^{\prime}$ & 0,11394 \\
\hline 2 & Roma & + & $4^{\circ} 35^{\prime} 30^{\prime \prime}$ & $316^{\circ} 15^{\prime}$ & 0,71679 \\
\hline 3 & Firenze & - & $6^{\circ} 32^{\prime} 15^{\prime \prime}$ & $321^{\circ} 38^{\prime}$ & 0,80498 \\
\hline 4 & Belgrado & + & $6^{\circ}+2^{\prime} 40^{\prime \prime}$ & $22^{\circ} 47^{\prime}$ & 0,80978 \\
\hline 5 & Zagabria & - & $7^{\circ} 20^{\prime} 30^{\prime \prime}$ & $355^{\circ} 35^{\prime}$ & 0,81496 \\
\hline 6 & Trieste & - & $7^{\circ} 18^{\prime} 40^{\prime \prime}$ & $343^{\circ} 43^{\prime}$ & 0,82434 \\
\hline 7 & Piacenza & - & $8^{\circ} 17^{\prime}$ & $322^{\circ} 32^{\prime}$ & 0,84407 \\
\hline 8 & Pavia & + & $8^{\circ} 36^{\prime} 36^{\prime \prime}$ & $320^{\circ} 38^{\prime}$ & 0,84906 \\
\hline 9 & Coira & 一 & $9^{\circ} 44^{\prime} 12^{\prime \prime}$ & $328^{\circ} 53^{\prime}$ & 0,86419 \\
\hline 10 & Strasburgo & $\ldots$ & $11^{\circ} 50^{\prime} 20^{\prime \prime}$ & $329 \circ 37^{\prime}$ & 0,90834 \\
\hline 11 & Varsavia & - & $13^{\circ} 51^{\prime} 51^{\prime \prime}$ & $10^{\circ} 53^{\prime}$ & 0,96569 \\
\hline 12 & Uccle & -- & $14^{\circ} 56^{\prime} 15^{\prime \prime}$ & $328^{\circ} 07^{\prime}$ & 1,00 \\
\hline 13 & Cartuya & + & $16^{\circ} 08^{\prime} 23^{\prime \prime}$ & $266^{\circ} 50^{\prime}$ & 1,05378 \\
\hline 14 & Eppsala & - & $21^{\circ} 10^{\prime} 30^{\prime \prime}$ & $1^{\circ} 18^{\prime}$ & 1,42815 \\
\hline 15 & EIsinki & -+ & $22^{\circ} 05^{\prime} 44^{\prime \prime}$ & $12^{\circ} 23^{\prime}$ & 1,47330 \\
\hline
\end{tabular}

rilevati dai sismogrammi. A questo proposito faccio osservare che pur essendo pochi i dati a disposizione (il terremoto non ha superato un'intensità intorno all'VIIIo della Mercalli) la disposizione delle stazioni è tale da consentire una notevole precisione nella determinazione grafica dei due piani tra loro ortogonali (Fig. 2). 
246

G. M. CAMELI

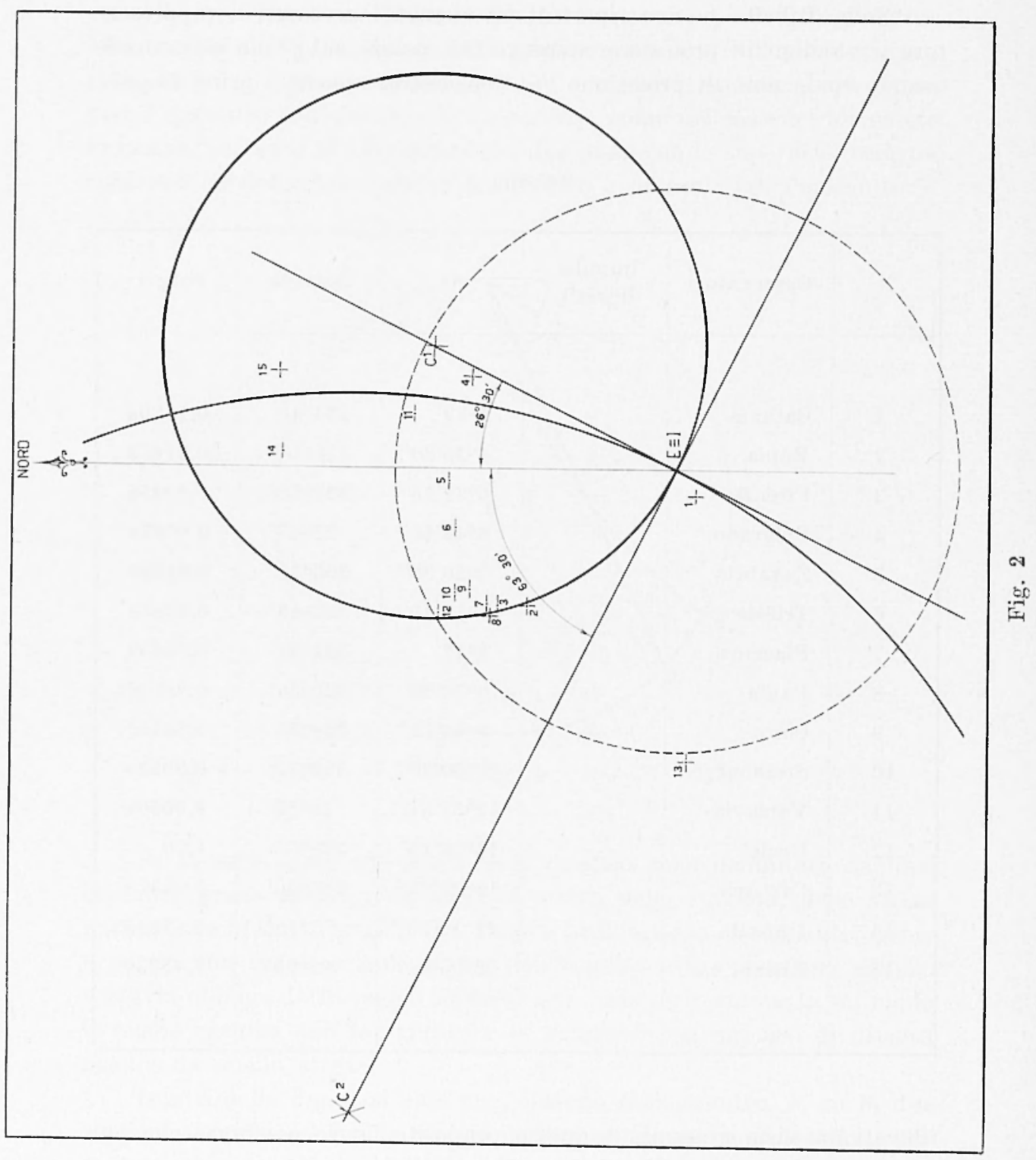


I due piani determinati hanno come direzione l'uno 63030' NW e l'altro $26^{\circ} 30^{\prime} \mathrm{NE}$. Come è noto la teoria matematica non consente di stabilire quale dei due piani sia l'effettivo piano di frattura; la sua determinazione è legata a cognizioni precedentemente acquisite (studio di altri terremoti) e alla situazione orografica, tettonica e gravimetrica.

\section{CoNSIDERAZIONI GEO-TETTONICHE SULLA DEPREsSIONe di CATANZARo.}

Esaminando la Carta geografica della Calabria si nota immediatamente il notevole restringimento che presenta la Penisola fra i due Golfi di Sant'Eufemia e di Squillace. In quel punto la larghezza massima della Calabria dal Mar Tirreno allo Jonio non raggiunge nemmeno i $30 \mathrm{~km}$; inoltre le montagne a Nord di Tiriolo e a Sud di Squillace raggiungono quasi tutte i $1000 \mathrm{~m}$ di altezza e in certi punti arrivano ai $2000 \mathrm{~m}$ mentre fra Tiriolo e Squillace non si hanno altezze maggiori ai $400 \mathrm{~m}$; nella zona compresa fra i due Golfi suddetti infatti si trovano altipiani alla quota media di $350 \mathrm{~m}$; mentre non vi è traccia delle creste appenniniche che si elevano a Sud e a Nord di questa zona.

Osservando la carta geologica di questa regione si nota che essa è un bacino di raccolta dei depositi clastici di età ponto-plio-quaternaria, mentre l'Appennino Calabrese è costituito da rocce cristalline antiche; pertanto a quell'eccezionale restringimento della Penisola corrisponde una vera e propria interruzione orografica e geologica dell'Appennino Calabrese mentre la conformazione topografica di una regione dovrebbe avere una stretta dipendenza con la struttura geologica della stessa. L'interruzione di una catena di montagne con cime abbastanza elevate, tenendo conto della natura rigida delle rocce cristalline costituenti l'Appennino Calabrese, può essere spiegata ammettendo l'azione di potenti fenomeni tettonici $\left({ }^{3}\right)$. D'altronde la completa interruzione della catena Appenninica fra i golfi di Squillace e Sant'Eufemia è dimostrata dal fatto che nella striscia considerata su una superficie di oltre $300 \mathrm{kmq}$. non si hanno resti di rocce cristalline che costituivano l'ossatura della catena montuosa.

L'ipotesi di rotture della crosta terrestre è quella che si presta meglio a spiegare questa particolare situazione e. con l'ipotesi di un sistema di faglie si giustifica la grandiosità del fenomeno.

Il piano di frattura con direzione $63^{\circ} 30^{\prime}$ W dall'epicentro del terremoto in esame perfettamente si accorderebbe con le precedenti considerazioni di ordine geotettonico anche in relazione al fatto che l'ipocentro è compreso nella crosta. 
A questo scopo è utile dare una descrizione sommaria della Geologia della regrione istmica compresa fra $\mathrm{i}$ due Golfi. Considerando Squillace e procedendo da W ad $E$ abbiamo graniti che si estendono a Sud fino a Soverato e ad W fino ad Amaroni e a Valle Fiorita. $\Lambda$ Nord abbiamo i graniti di Catanzaro che si estendono verso Sud dove formano il massiccio della Sila e verso IV fino oltre Tiriolo anche se con caratteristiche variate; compaiono quindi filladi assieme a masse di serpentine che si estendono fin oltre Gizzeria a Castiglione; da Castiglione al mare affiorano colline di argille e molasse del Miocene superiore. Su queste piccole colline ed anche presso Marcellinara si hanno delle masse di calcare siliceo e di gesso che rappresentano la zona gessosa-solfifera. Nella parte intermedia fra i terreni cristallini già descritti si hanno degli altipiani genera.mente alla quota di $350 \mathrm{~m}$ di terreni pliocenici e quaternari. La pianura del Golfo di Sant'Eufemia è una grande estensione di alluvionale recente ed è probabile che il Golfo si protraesse molto di più entro la terra. Dalla fiumara di Nicastro fino al Golfo di Squillace si ha una serie completa dal Pliocene inferiore al Quaternario. Le sabbie gialle, le argille azzurre più o meno conglomerate del Quaternario dimostrano una quasi completa orizzontalità degli strati.

Il distacco brusco che aveva messo in comunicazione i due mari doveva essersi verificato alla fine del Miocene. Nel Miocene Medio sarebbe avvenuta una fase di corrugamento con assi a direzione N-S seguita dalla formazione di flessure con assi E-W (asse Catanzaro-Nicastro) e con assi ESE-WNW (zona di Amantea-Catanzaro). Tra la fine del Miocene ed il Pliocene superiore sarebbero comparse faglie normali; una di queste secondo il Cortese andrebbe dal Capo Sovero alla punta di Staletti quasi perfettamente rettilinea e passerebbe sotto Maida presso Cortale e presso Squillace. Essa sarebbe individuata da varie particolarità della fisionomia topografica della regione considerata; Capo Sovero si protende di molto in mare rispetto alla linea di costa del Golfo, e ciò doveva essere molto più visibile prima della formazione di quella grande plaga di terreni alluvionali. Presso Maida e Jacurso si hanno ripide pendici di rocce dioritiche di vario tipo contro le quali urtano le marne biamche a foraminiferi del Pliocene con un dislivello di $350 \mathrm{~m}$ tra la cima delle pendici e la linea di contatto del Pliocene. Presso Capo Copanello e la punta di Stalettì ci sono dei veri appicchi di graniti che sporgono in mare contrastando nettamente con le spiagge e collinette che formano il Golfo di Squillace. La direzione di questa faglia coincide, a volte singolarmente, con la traccia del piano di frattura determinato dal terremoto in essime. 
Durante il Pliocene la sedimentazione proseguì nel braccio di mare di Catanzaro-Nicastro. Il bacino della piana di Sibari è l'area più estesamente ricoperta da depositi dell'olocene e ad essa segue come importanza la pianura di Sant'Eufemia. Varie linee tettoniche si intersecano sotto la pianura di Sibari ed anche la piana di Sant'Eufemia è tettonicamente attiva come pure il territorio di Catanzaro-Nicastro (*).

\section{CONSIDERAZIONI GRAVIMETRICHE.}

Per ciò che riguarda la situazione gravimetrica riportiamo i risultati ottenuti da Ciani A.-Gantar C.-Morelli C. ( $\left.{ }^{4}\right)$.

Nello Jonio le misure in mare danno indicazioni sulla continuazione verso SE della grande fascia di anomalie negative corrispondenti alla fossa sedimentaria periappenninica esterna (Golfo di Sibari). Più a Sud la costa strapiomba nel mare per cui l'isobata di $-200 \mathrm{~m}$ viene raggiunta a breve distanza dalla costa stessa, perciò il contributo delle misure di gravitì in mare non può essere determinante. Comunque esse consentono di intravedere sul lato dello Jonio la continuazione della fascia di anomalie negative dell'arco periappenninico esterno (Golfi di Sibari, Squillace, Locri) mentre i tre speroni di C. Rizzuto, C. Stilo, e C. Spartivento corrispondono ad anomalie fortemente positive; è interessante notale che la depressione del Golfo di Squillace si continua gravimetricamente in quella di Sant'Eufemia: ciò è indice che essa ha origine da una sinclinale degli strati più densi (Fig. 3).

L'arco calabro-siciliano è attualmente in via di deformazione. I due bordi, nella regione di Sibari ed in quella di Cetraro, sono in via di affossamento come la fossa di Catanzaro. La forte anomalia gravimetrica positiva della regione di Cetraro-Amantea raggiunge i +100 mgal e +90 a Cetraro. Sono possibili più soluzioni: dal punto di vista geologico è ammissibile l'ipotesi che la crosta della catena costiera non sia una crosta sialica di tipo normale ma una crosta di tipo intermedio. Questa ipotesi sarebbe confortata dal valore della velocità ottenuto per le $P$ e $S$ da Valle per il terremoto del Golfo di Squillace $(6,09 \pm 0,26$ e $3,63 \pm 0,09 \mathrm{~km} / \mathrm{sec}$ rispettivamente).

$\left({ }^{*}\right)$ V. Carta geologica e tecnica della Calabria al 25.000 eseguita dal C.A.R. per conto della Cassa per il Mezzogiomo, 1961.

Beveo E., Tentativo di sintesi tettonica dell'Italia peninsulare ed insulave "Boll. Soc. (reol. Italiana ", LXVIII (1949). 
Nella regione di Sibari (5) è assai probabile che questa crosta sopporti una serie sedimentaria terziaria di 4 o $5 \mathrm{~km}$. Per quanto riguarda la fossa sedimentaria di Catanzaro gli spessori massimi dedotti in superficie dai geologi per le serie complete dei sedimenti terziari e quater-

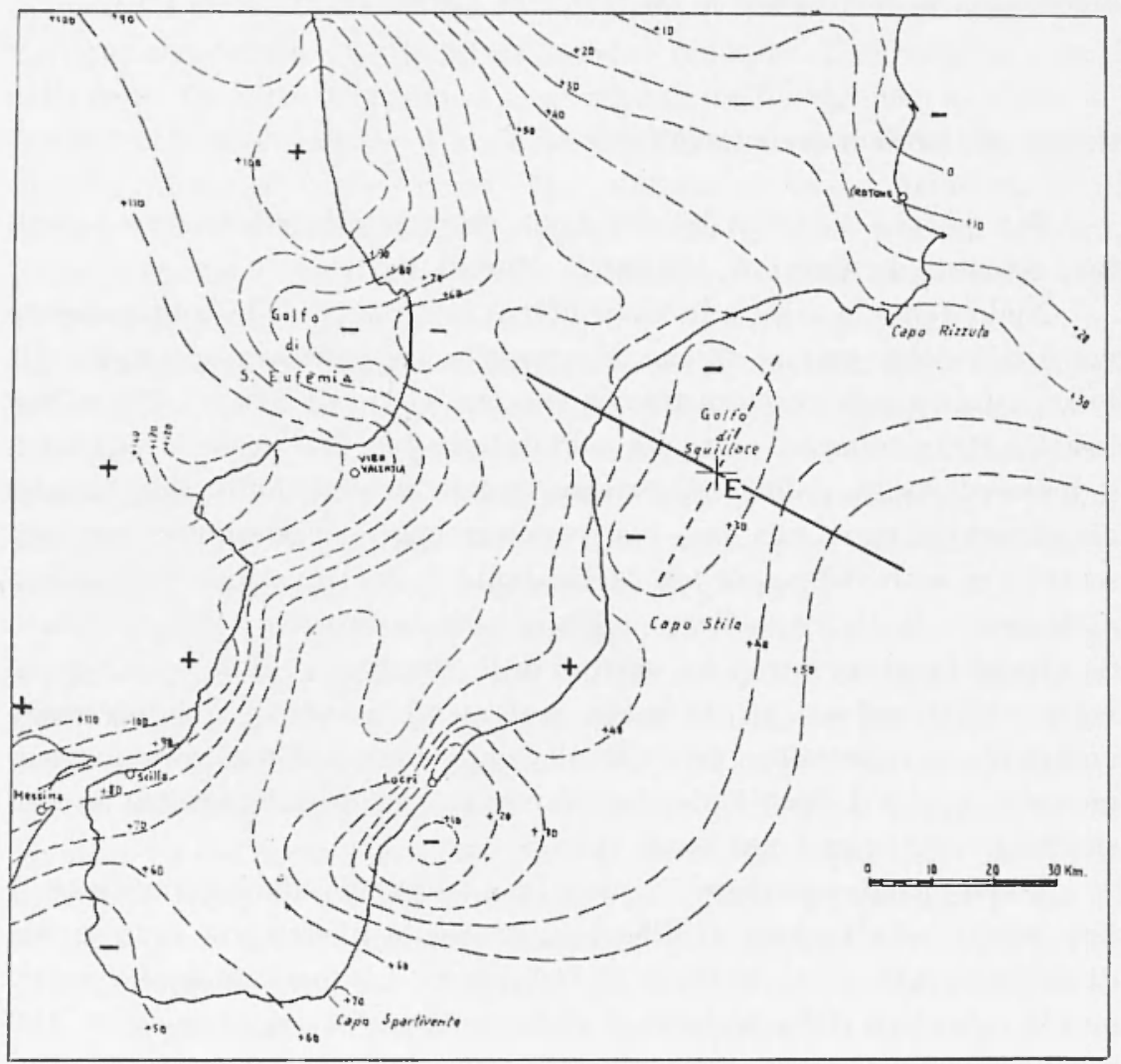

Fig. 3-Carta gravimetrica della Calabria e piano di frattura del terremoto del golfo di Squillace.

nari sarebbe al più di $1000 \mathrm{~m}$; è probabile quindi che la crosta sopporti una serie sedimentaria terziaria di $2-3 \mathrm{~km}$.

La fossa di Catanzaro offre anche un'anomalia magnetica trasversale E-W dovuta probabilmente all'affossamento ponto-plio-quaternario; questo interrompe perciò la continuità delle anomalie positive della regione costiera $\left(^{5}\right)$. 
STORIA SISMICA DELLA REGIONE COMPRESA TRA I GOLFI DI SANT'EUFEMIA E Squillace.

La scossa dell'11 Maggio 1947 con epicentro nel Golfo di Squillace fu valutata di VIIIo nella zona più colpita che comprende $i$ paesi di Borgia, Squillace, Soverato, Badolato. Fu avvertita di VIIo a Catanzaro, di VIo a Sambiase, di Vo ad Amantea e Tropea. Il terremoto fu avvertito in tutta la Calabria meridionale fino a Reggio ed anche in Sicilia, a Nord fu avvertito fino a Cosenza.

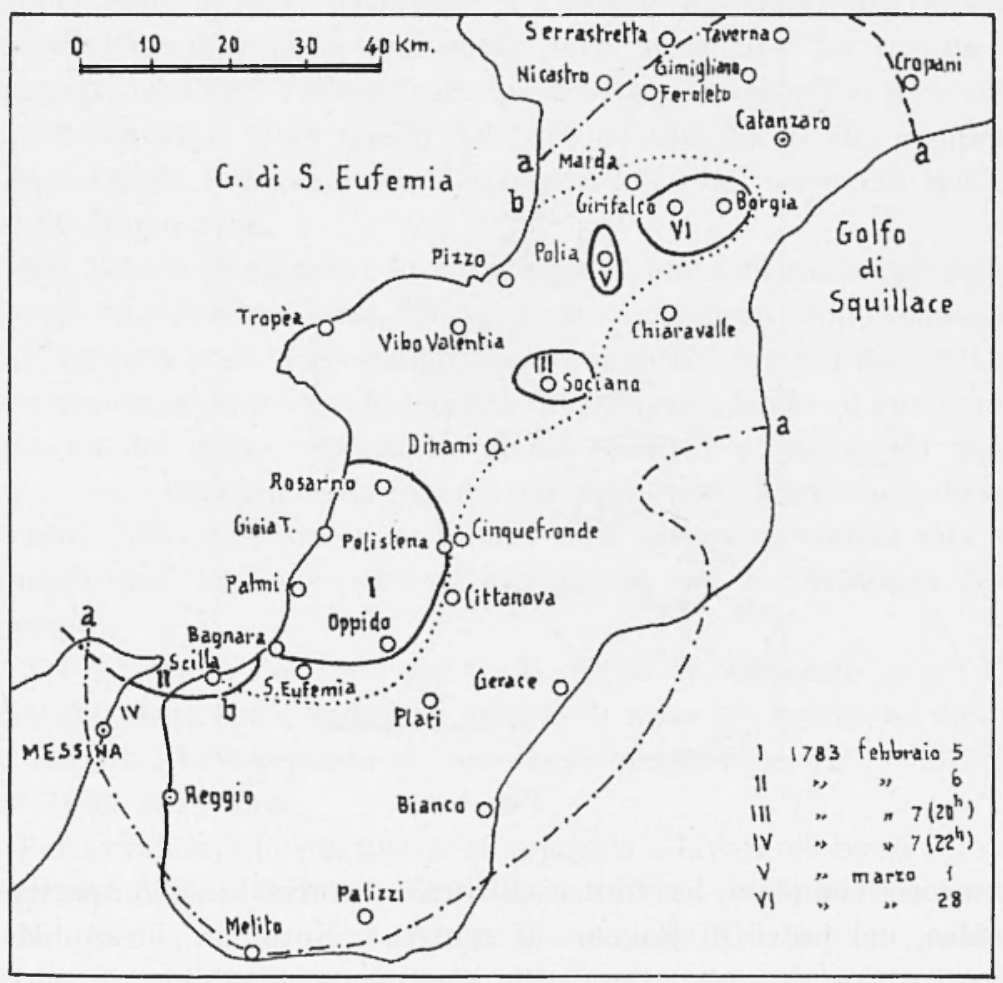

Fig. 4

La regione istmica costituisce una zona sismica assai attiva che spesso ha dato luogo a dei massimi d'intensità veramente disastrosi per esempio quelli del 1659, 1783 e 1905 per citare solamente i maggiori. L'area mesosismica del parossismo del 1659 è ristretta, crociforme: un suo braccio il $\mathbf{N}$-S si estende da Castelmonardo (Filadelfia) a Gero- 
carue; quello E-W dai pressi di Vibo Valentia (Nonteleone) a Chiaravalle. Nell'estremo braccio settentrionale include Polia e Poliolo, epicentro del disastroso terremoto del $1^{\circ}$ Marzo 1783; nella sezione meridionale si trovano Soriano. Sorianello e Gerocarne colpita duramente dal parossismo del 1783.

Per quanto riguarda l'area mesosismica del terremoto calabro del 1783 riporto un sismo-cartogramma del Baratta $\left(^{6}\right)$ (Fig. 4). L'area della

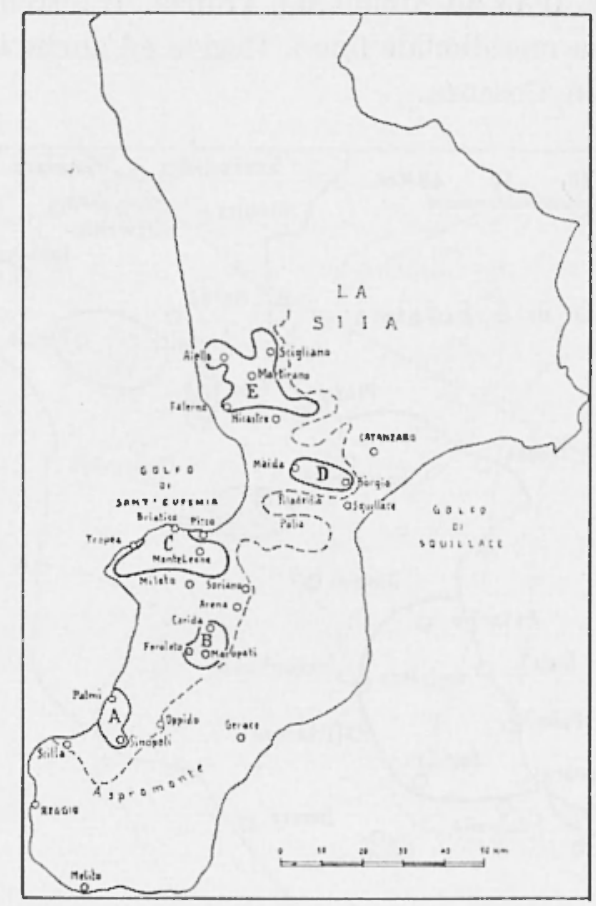

Fig. 5

distruzione completa ha forma ellittica e, correndo sullo spartiacque tirrenico, dai pressi di Bagnara si spinge a Nord fin presso Maida, Jacurso e Cortale (a-a); l'area che comprende gli abitati in massima parte distrutti o inabitabili giunge a Nord a Feroleto, Taverna e Soveria e a Sud fino a Melito e Palizzi. I massimi che si verificarono furono 6 secondo il Baratta e il Vo e il VIo furono quelli che interessarono particolarmente la regione presa in esame. L'area epicentrale del Vo (10 Marzo) si trova nel bacino del Reschia, affluente dell'Angitola o comprende Polia e Poliolo che ebbero distrutte gran parte delle loro abitazioni; nel VIo massimo (28 Marzo) l'area epicentrale si sposta a Girifalco- 
Borgia-Maida nella regione istmica. I vari massimi irraggiarono da epicentri ben identificati di nota attività.

Per la distribuzione delle intensità del terremoto calabro dell'8 Settembre 1905 riporto il sismo-cartogramma dal Baratta (Fig. 5).

Si osserva che la zona più gravemente colpita risulta assai espansa e si estende lungo la marina da Scilla a Belvedere (150 km) e l'isosista che la delimita presenta un irregolarissimo decorso con bruschi cambiamenti di direzione oppure con ampie flessuosità.

Entro questa zona si distinguono alcune sezioni nelle quali i danni inferti dall'azione distruttrice del parossismo sismico risultano assai maggiori. Sono sette e si trovano a notevole distanza l'una dall'altra irregolarmente distribuite nell'ampia zona accennata. La sezione $C$ è sviluppata nel Capo Vaticano; da questa zona irraggiarono pure alcuni massimi secondari quali quello del 1869 e 1886. Isa $D$ che comprende Maida e Borgia si sovrappone alla mesosismica dei terremoti del 1626 e del 28 Marzo 1783.

Nel 1783 il parossismo fu costituito da un accentuato periodo sismico prolungatosi per lungo tempo: in tre anni si contarono 1650 scosse; il loro numero andò decrescendo ma con parecchi bruschi aumenti, riusciti maggiormente intensi in località diverse estendendo ed aumentando la gravità dei danni. Spesso in alcuni violenti e prolungati periodi sismici i varii massimi catastrofici si manifestarono ad una certa distanza di tempo l'uno dall'altro e colpirono zone diverse attuando una vera trasmigrazione degli epicentri ed estendendo così la rovinosità del fenomeno.

Nel terremoto del 1905 per G. B. Rizzo (v. Cavasino A.) (7) l'epicentro sarebbe unico e cadrebbe proprio in mare nel mezzo del Golfo di Sant'Eufemia in un punto di coordinate geografiche $\varphi_{0}=38^{\circ} 50^{\prime} \mathrm{N}$, $\lambda_{o}=16^{\circ} 6^{\prime} \mathrm{E}$ (Fig. 6).

Per avvalorare la sua ipotesi si appoggia a lavori del Suess “ il quale ha dimostrato l'esistenza di una linea di frattura periferica che percorre le Calabria e la Sicilia a guisa di un grande arco circolare che, partendo da Cosenza, per Rogliano, Girifalco, Terranova, Oppido, Santa Cristina, Regrrio taglia la Sicilia da Ali a Bronte a Capizzi, avendo il suo centro nella regione delle Isole Lipari.

Oltre a questa frattura principale ve ne sono altre press'a poco rettilinee le quali irradiano a guisa di ventaglio dal centro della prima intersecando la Calabria e la Sicilia: una, fra le altre presso Rende da SW a NE, un'altra dal Golfo di Sant'Eufemia a quello di Squillace. Nelle regioni dove ciascuna delle fratture radiali incontra la frattura 
periferica, la roceia primaria presenta la massima libertà di movimento e quando avviene una scossa, qui si hanno come delle regioni ventrali di immani lamine elastiche.

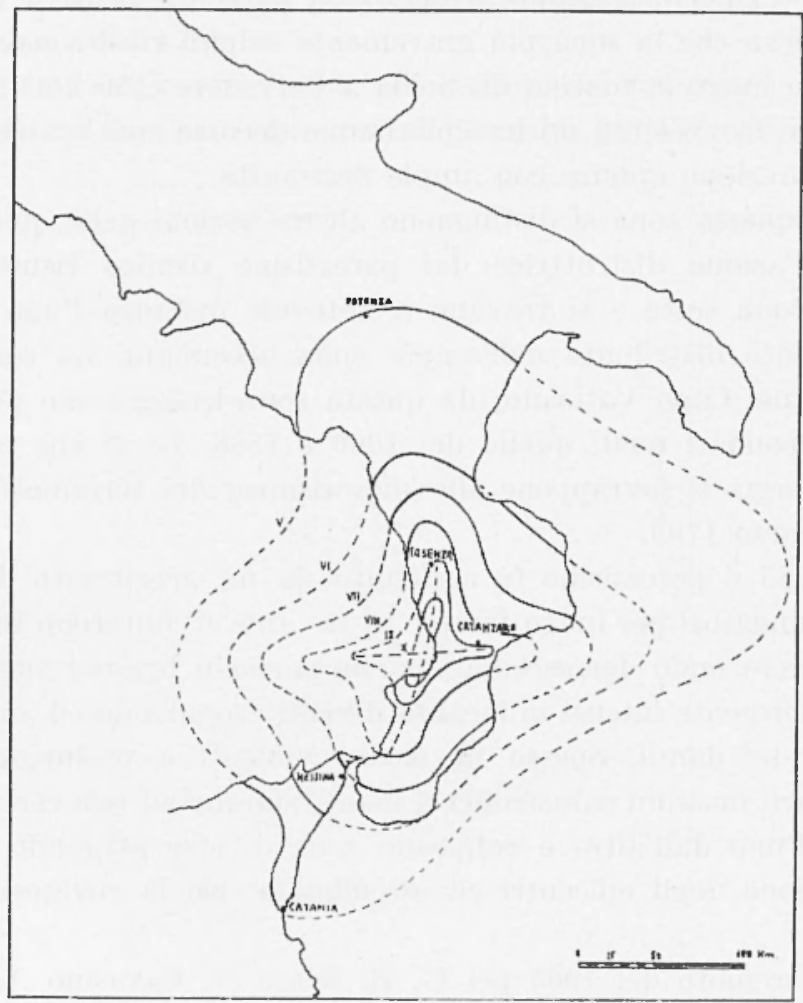

Fig. 6 - Isosisme del terremoto calabro dell'8 Settembre 1905 (secondo G. B. Rizzo).

Qui sono più intensi i movimenti sismici e soltanto le diverse condizioni dei materiali sedimentari, la varia inclinazione e delle rocee primarie rispetto all'orizzonte, e specialmente il modo con cui sono gettate le fondamenta e sono eseguite le costruzioni degli edifici, possono determinare la particolare distribuzione dei damni prodotti dal terremoto ".

Ora dall'esame delle distribuzioni dei movimenti del suolo del terremoto del settembre 1905 il Rizzo è costretto ad ammettere l'esistenza di un'altra linea di frattura nella concavità di quella del Suess, la quale partendo da Ajello attraversa il Golfo di Sant'Eufemia e raggiunge i territori di Briatico, Monteleone, Cessaniti; infatti lungo questa linea si 
ebbero i momenti più intensi e le maggriori rovine. Egli pone perciò il suo epicentro nell'incontro di questa seconda linea di frattura periferica con la frattura radiale passante per il Golfo di Sant'Eufemia.

Il $1^{\circ}$ Agosto 1910 a Borgia in provincia di Catanzaro fu avvertita una scossa di VIo. Il terremoto fu sentito in quasi tutta la Calabria e Sicilia Orientale. Il 7 Novembre 1913 fu avvertita a Filadelfia (Catanzaro) una scossa molto forte che provoco caduta di calcinacci e qualche lesione leggera negli edifici meno solidi. La scossa fu forte a Borgia e a Pizzo; mediocre a Badolato, Catanzaro, Gasperina, Martirano, Mileto, Sambiase e Tiriolo; leggera a Mongiana, Petilia Policastro, Soveria Simeri, Tropea e Laureana di Borello interessando cosi tutta la larghezza della Calabria dall'uno all'altro mare attorno alla Stretta di Catanzaro e tra Petilia Policastro e Laureana di Borello in direzione quasi longitudinale.

Il 13 Giugno 1913 a Bagni di Sambiase fu avvertita una scossa molto forte preceduta da un fortissimo rombo; si ebbe un aumento di portata delle acque termali che divennero fangose. Si trattò, ad ogni modo, di un fenomeno strettamente locale poiché la scossa fu avvertita mediocremente a Sambiase e Nicastro e debolmente a Feroleto, Santa Eufemia e Tiriolo; non si ebbero repliche.

Con le due scosse dei giorni 6 e 7 Gennaio 1929 culminó il periodo sismico del Catanzarese che era iniziato il 27 Dicembre del 1928. Le due scosse raggiunsero il VIo a Borgia. I paesi che subirono qualche lieve danno per le scosse più forti furono Borgia, Cortale, Girifalco. Le due scosse del 6 e 7 Gennaio furono avvertite distintamente anche a Catanzaro Marina, San Floro, Curinga, Maida, Nicastro. L'epicentro le cui coordinate geografiche sono

$$
\begin{aligned}
& \varphi_{0}=38^{\circ} 50^{\prime} \quad \mathrm{N} \\
& \lambda_{0}=16^{\circ} 29^{\prime}
\end{aligned}
$$

è compreso tra Borgia e Girifalco. La propagazione sensibile non andò oltre i $24 \mathrm{~km}$ attorno a questo punto, la zona macrosismica ebbe quindi un'estensione di circa $650 \mathrm{kmq}$.

Il 9 Ottobre 1930 una scossa del VIo colpì Crotone producendo lievi lesioni in qualche fabbricato e cadute di calcinacci. La scossa fu avvertita distintamente su tutto il litorale jonico della provincia di Catanzaro e debolmente fino a Tatiano e Messina dimodoché tutto fa supporre che l'epicentro si trovi nel mare Jonio. Circa un quarto d'ora dopo si avvertì una forte replica, poi più nulla. 
La città di Catanzaro, dal punto di vista sismico, è sottoposta sia all'azione del distretto sismico del Cosentino, zona di violentissimi terremoti, sia ai centri sismici intomo a Nicastro e infine a quelli che si irradiano dalla sua marina; per questo motivo non si riusci a stabilire con esattezza la provenienza delle scosse sentite nell'agosto 1821, che fecero rovinare aleme case nei pressi di Catanzaro causando diverse vittime.

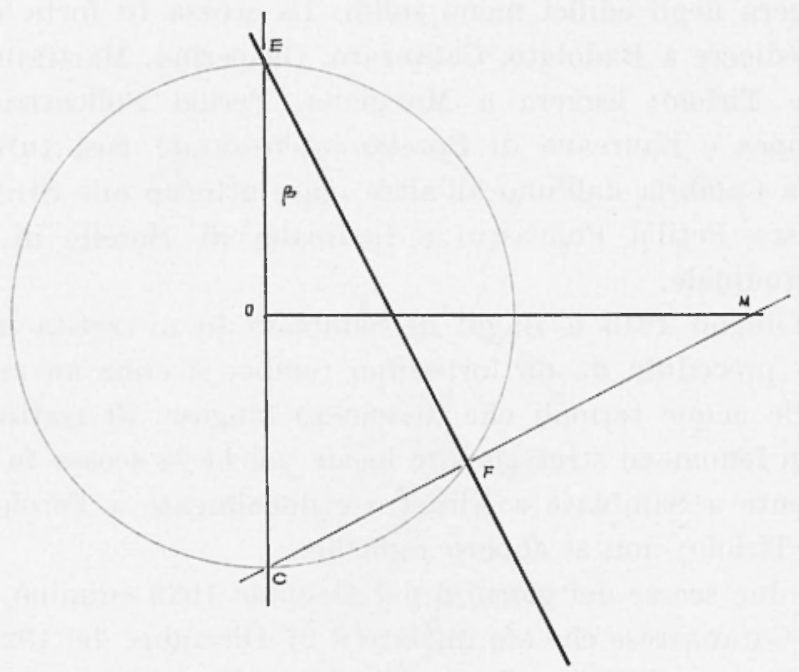

Fig. 7 - Inclinazione del piano di frattura rispetto all'asse terrestre per l'epicentro.

Tra Nicastro e Cosenza è solo ricordato il terremoto del 1638. Si sa che dai dintorni di Nieastro si sono irraggiati vari teremoti abbastanza localizzati come quello rovinoso del 1609 e quello fortissimo del 1728. La posizione di Nicastro è chiarita dalla scossa fortissima che il 27 Magrgio 191 . fu molto intensa a Bagni di S. Biase.

Il versante jonico della Calabria a Sud della strozzatura esistente tra i Golfi di Squillace e Sant'Eufemia ha sismicità in genere non elevata, con parechi centri che hamno dato luogo a manifestazioni localizzate anche se intense. Lungo la marina sottostante a Montauro esiste un centro secondario di attività fissato dalla scossa del 7 Marzo 1783 sentita lungo quel litorale. Il paese di Badolato fu interessato da due scosse avvertite il 19 e 22 Giugno dello stesso amno. $A$ Stilo il 12 Dicembre 1679 fu sentita una scossa molto forte di cui mancano però notizie riguardanti altre località (7). 


\section{Conct.usioni.}

I. dati del rilievo gravimetrico e le condizioni geotettoniche della regione istmica mi hamno indotto a scegliere, tra i due piani ottenuti

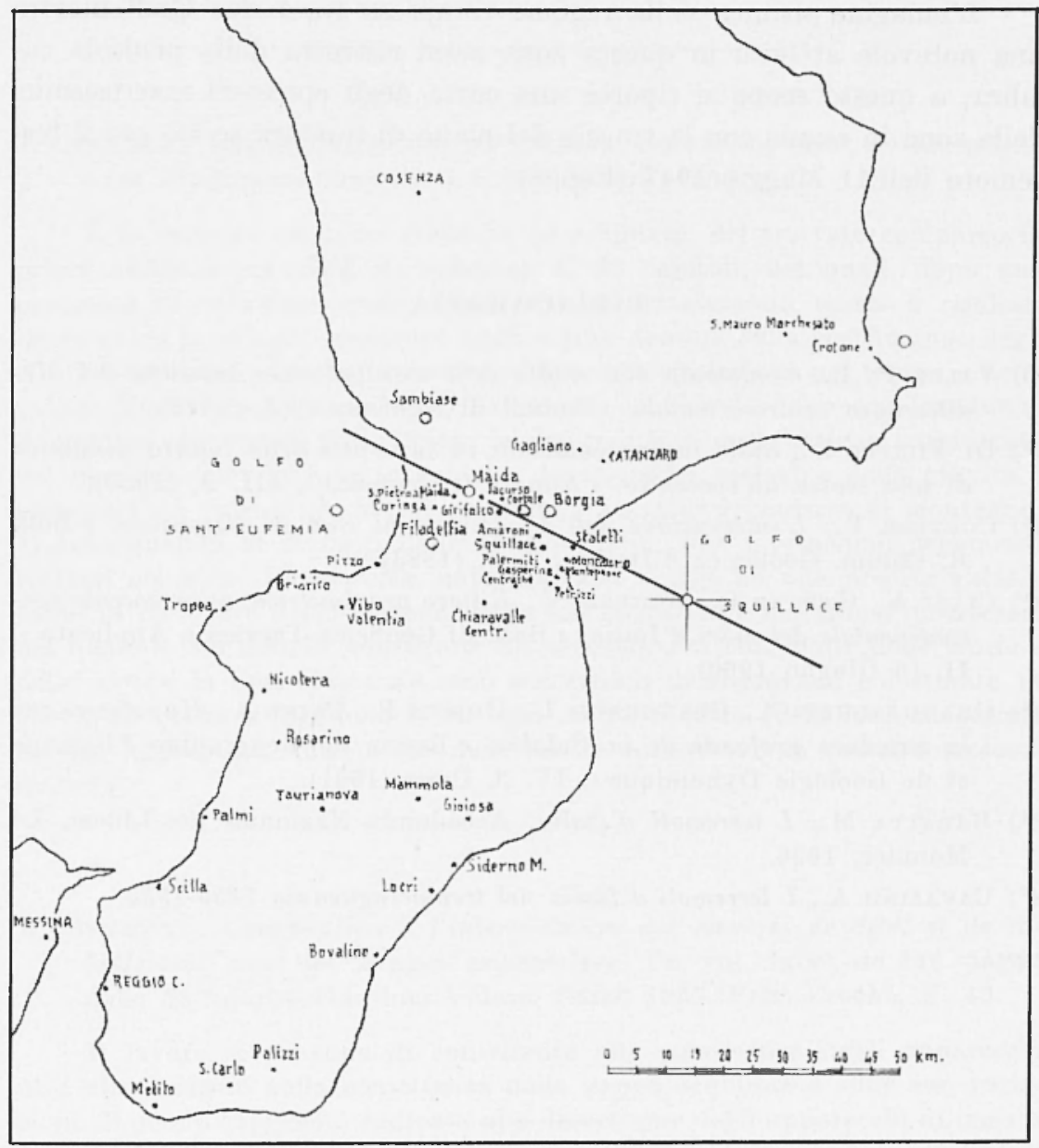

Fig. 8 - Iipicentri macrosismici della regione istmica (O) e piano di fratture del terremoto del Golfo di Squillace.

della teoria matematica, quello di direzione 63030' $\mathrm{W}$; l'inclinazione del piano di fuattura rispetto al raggio terrestre per l'epicentro è $\beta=27^{\circ}$.

Infatti tracciato $m$ cerchio corrispondente ad ma sezione meridiana, si riporta sulla traccia del piano equatoriale a partire dal centro 
il diametro del cerchio unendo poi l'estremo di questo segmento con il centro di proiezione (Fig. 7). La corda che unisce l'epicentro con l'intersezione della retta proiettante con il cerchio meridiano rappresenta la traccia del piano e l'angolo che essa forma con il raggio per $D$, l'angolo d'inclinazione cercato.

L'indagine sismica della regione compresa fra i due Golfi mostra una notevole attività in questa zona assai ristretta della penisola calabra; a questo scopo si riporta una carta degli epicentri macrosismici della zona in esame con la traccia del piano di frattura scelto per il terremoto dell'11 Maggio 1947 (Fig. 8).

\section{BIBIIOGRAFIA}

(1) VALLE P. E., Contributo allo studio delle caratteristiche sismiche del Mediterraneo centro-orientale. "Annali di Geofisica", 2, (1948).

$\left({ }^{2}\right)$ Di Filippo D., Sulla rappresentazione in superficie della natura dinamica di una scossa all'ipocentro. "Annali di Geofisica", III, 2, (1950).

$\left({ }^{3}\right)$ Cortese E., L'interruzione dell'Appennino al Sud di Catanzaro. "Boll. R. Comm. Geologica d'Italia ", 7, 8, (1883).

(') Ciani A., Gantar C., Morelli C., Rilievo gravimetrico dello zocoolo epicontinentale dei mari d'Italia. "Boll. di Geofisica 'Teorica e Applicata", II, (6 Giugno 1960).

(5) Grandjaquet CI., Glangeaud L., Dubois R., Catre A., Hypotheses sur la structure profonde de la Calabre. "Revue de Geographie Physique et de Geologie Dynamique ", IV, 3, Paris (1961).

$\left({ }^{8}\right)$ Baratta M., I terremoti d'Italia. Accademia Nazionale dei Lincei, Le Momnier, 1936.

(') Cavasino A., I terremoti d'Italia nel trentacinquennio 1899-1933. 